\title{
A new technique and results of measurements of dielectric and magnetic properties of loss dielectrics for vacuum tubes
}

\author{
Evgeniia Bogomolova, Anatoly Galdetskiy", Vladimir Vasilev, and Vladimir Alekseenkov \\ JSC “RPC “Istok” named after Shokin”, 141190 Fryazino, Russian Federation
}

\begin{abstract}
A technique for measuring dielectric and magnetic properties of materials by the waveguide method is proposed in which the error, associated with the presence of gaps between the sample and the waveguide wall is eliminated by comparison with the results of numerical analysis of a 3D model the dielectric characteristics of which are optimized in order to achieve the best agreement between the calculated and experimental data.
\end{abstract}

\section{Introduction}

Defining dielectric properties of absorbing materials is an important part in microwave electronics. This information is critical in the development of many microwave devices. The problem of measuring is not a new one, a great number of procedures for measuring dielectric and magnetic properties of the substance in microwave [1-3] have been proposed. However, this diversity suggests that this problem has not been solved yet.

In this work the task was to determine four characteristics of the absorbing ceramics using the waveguide measurement method. It is evident that to determine the four parameters $\left(\varepsilon^{\prime}, \varepsilon^{\prime \prime}, \mu^{\prime}, \mu^{\prime \prime}\right)$ four values must be measured.

The waveguide method allows to measure different materials (including those with high absorption) in a very wide range of wavelengths (from 10 to $1-0.5 \mathrm{~cm}$ ). The essence of the method is that S-parameters of the waveguide segment with the sample placed there are measured, and as a result of processing these data, the material parameters are determined [4]. At the same time the simplest Nicolson-Ross-Weir (NRW) algorithm [5-6] can produce a significant error due to the influence of a badly-controlled gap between the measured sample and the waveguide wall.

To solve this problem the NRW algorithm and a correction formula were proposed, the latter takes into account the gap between the sample and the waveguide (1) [7].

$$
\varepsilon_{S}=\frac{\varepsilon_{N R W}(b-\Delta b)}{b-\Delta b \varepsilon_{N R W}}
$$

where, $\varepsilon_{S}$ is a relative permittivity of the sample material, $\varepsilon_{N R W}$ is a measured relative permittivity, calculated using the NRW algorithm, $b$ is the height of the waveguide, $\Delta b$ is the height of the gap.

* Corresponding author: galdetskiy@istokmw.ru 
A)
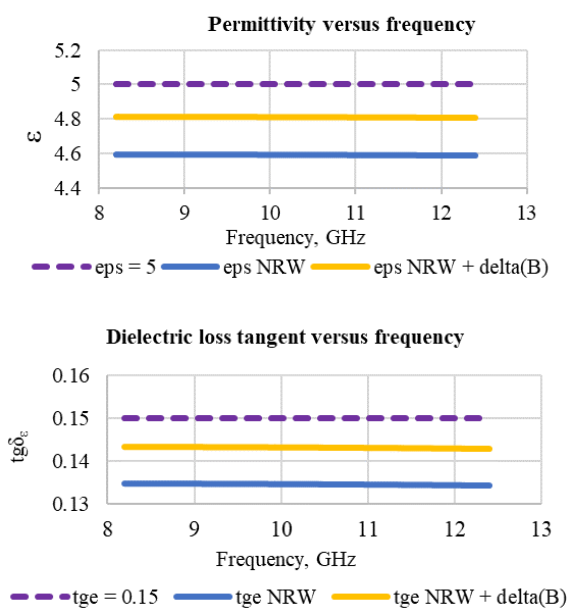

B)
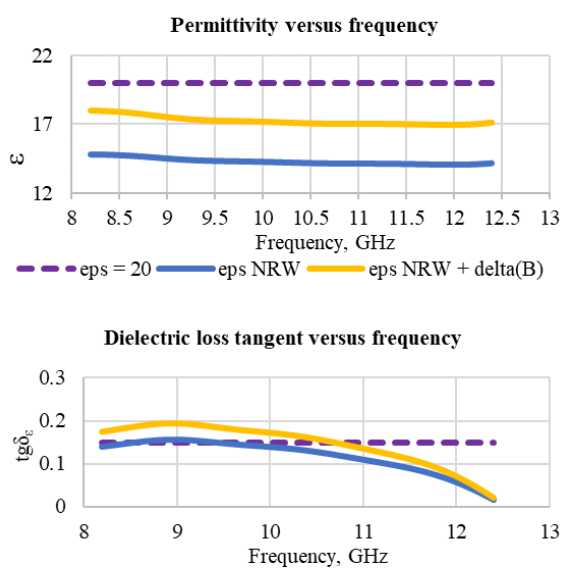

$\longrightarrow$ tge $=0.15 \longrightarrow$ tge NRW $\longrightarrow$ tge NRW + delta(B)

C)
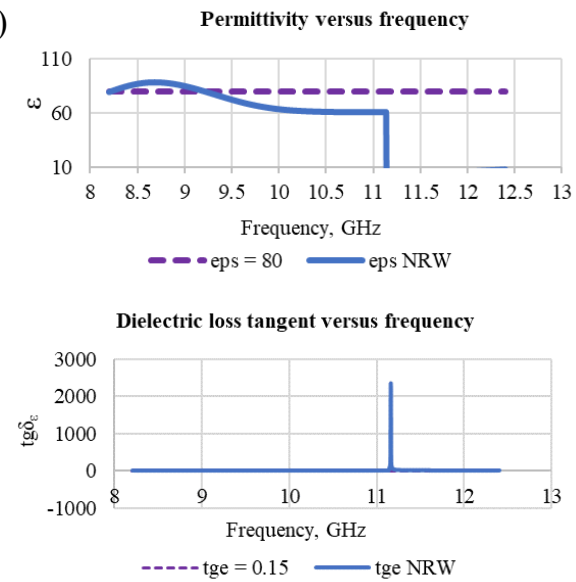

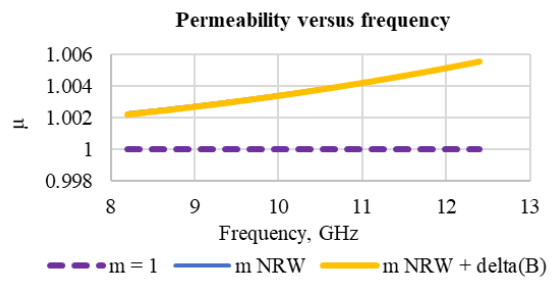

Magnetic loss tangent versus frequency

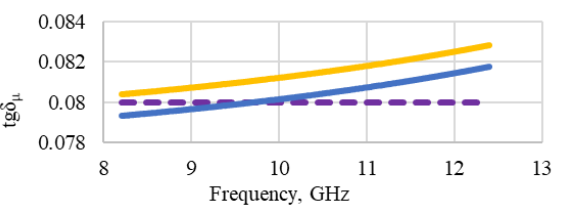

$\longrightarrow$ - $-\operatorname{tgm}=0.08 \longrightarrow \operatorname{tgm} N$ tgm NRW $+\operatorname{delta(B)}$

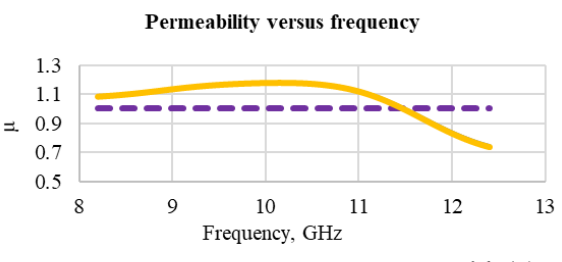

$-m \mathrm{~m}=1 \longrightarrow \mathrm{mRW}$ NRW $+\operatorname{delta}(\mathrm{B})$

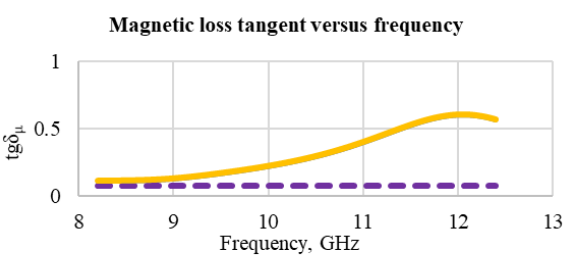

$-\boldsymbol{\bullet} \operatorname{tgm}=0.08 \longrightarrow \operatorname{tgm} \mathrm{NRW} \longrightarrow \operatorname{tgm~NRW}+\operatorname{delta}(\mathrm{B})$
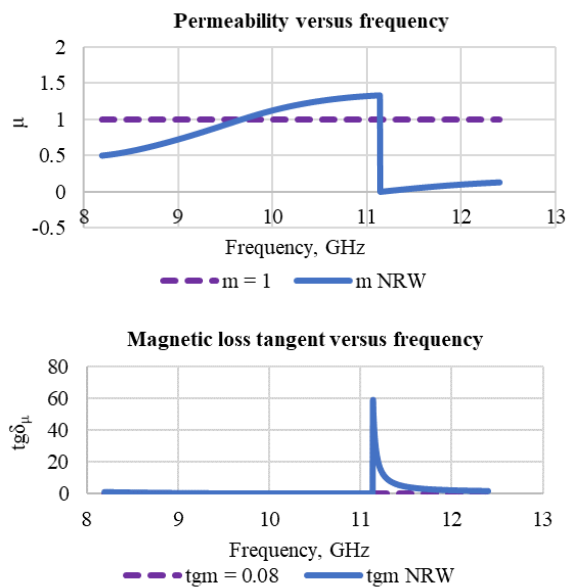

Fig. 1. Dielectric and magnetic characteristics, calculated with corrected and non-corrected NRW algorithm for three reference material samples with the length of $3 \mathrm{~mm}$, the gap of $161 \mathrm{um}, \mu=1$, $\operatorname{tg} \delta_{\varepsilon}=0.15, \operatorname{tg} \delta_{\mu}=0.08$ and $\varepsilon=5(\mathrm{~A}), \varepsilon=20(\mathrm{~B}), \varepsilon=80(\mathrm{C})$. 
A study to determine the material characteristics, corrected by formula (1), by NRW algorithm showed that for dielectrics with a value of $\varepsilon \leq 5$ the relative error $\varepsilon$ does not exceed $4 \%$, and at $\varepsilon \geq 20$ the relative error becomes large, which is associated with a distortion of the field near the ends of the sample. Figure 1 shows the curves of dependences of permittivity and permeability, dielectric and magnetic loss tangents versus frequency where the gap height between the test sample and the narrow waveguide wall is $\Delta \mathrm{b}=131 \mathrm{um}$, and the sample thickness is $3 \mathrm{~mm}$.

It is evident that the greater the height of the $\Delta b$ gap is, the greater is the relative error for the same material. From the above it follows that this method of determining the parameters of the material has limitations. In operation the studied dielectrics have a high permittivity and high losses, therefore, to determine their electromagnetic characteristics in a wide frequency range, it is necessary to develop a new measurement method free from the above disadvantages.

\section{Determination of material parameters by comparing measurements with the design characteristics of the model}

The proposed measurement technique is focused on determining permittivity and permeability, the tangent of dielectric and magnetic losses through the measured values of the module and phase $S_{11}, S_{21}$ of the waveguide segment with the sample.

Description of the technique:

- Accurate measurement of the test sample dimensions

- Measurement of S-parameters by a waveguide insert with the test sample

- Creating a measurement cell model in the electrodynamic simulation program with parameterized frequency-dependent values of $\varepsilon^{\prime}, \varepsilon^{\prime \prime}, \mu^{\prime}, \mu^{\prime \prime}$ (fig.2)

- By optimizing the values of $\varepsilon^{\prime}, \varepsilon^{\prime \prime}, \mu^{\prime}, \mu^{\prime \prime}$ in the frequency band adjust the calculated and measured S-parameters curves, determine the desired values of electrodynamic characteristics of the material.

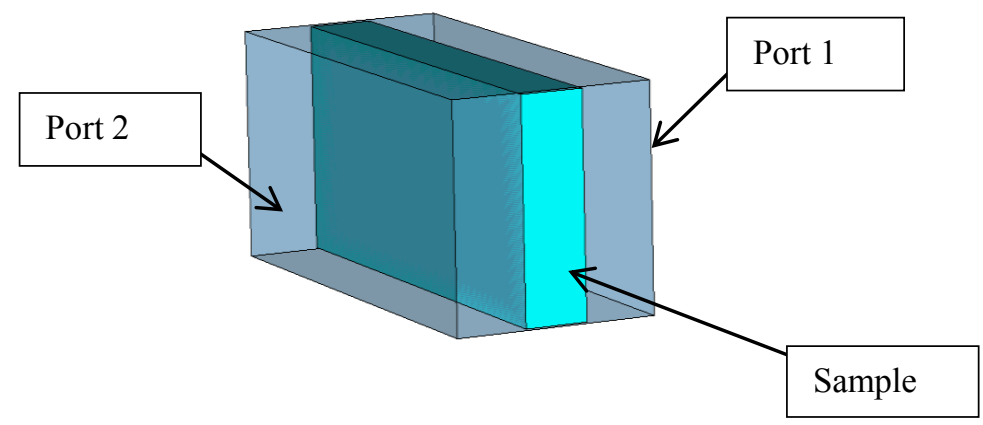

Fig. 2. 3D-model of the measuring line.

Figures 3-4 show the module of the reflection and transmission coefficients of AHMКХ2 and ПКМ-35Ж materials in the frequency range of 8-12 GHz, and Figures 5-6 show electrodynamic characteristics of these materials, respectively, determined by the new method.

The modeling showed that at the values of the sample permittivity in the range of $20-$ 25 the optimization procedure reduces to the true value of dielectric parameters regardless of the starting values of these parameters. Optimization was carried out by Nelder Mead Simplex Algorithm method in CST Studio program [8]. 


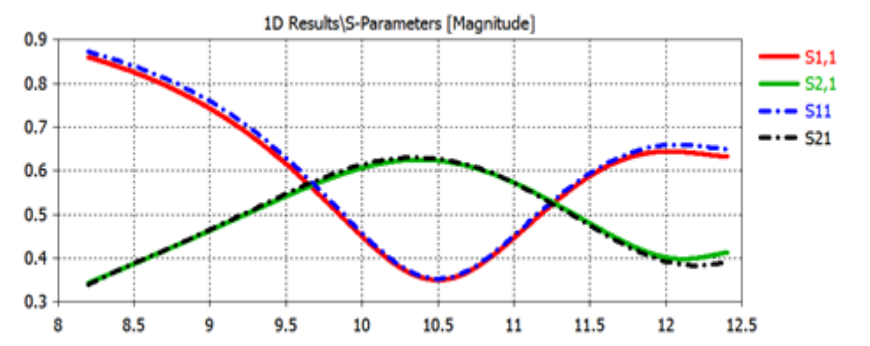

Fig. 3. The module of the reflection and transmission coefficients of the AH-MKX2 material samples: solid curves - calculated, dash-dotted curves - measured.

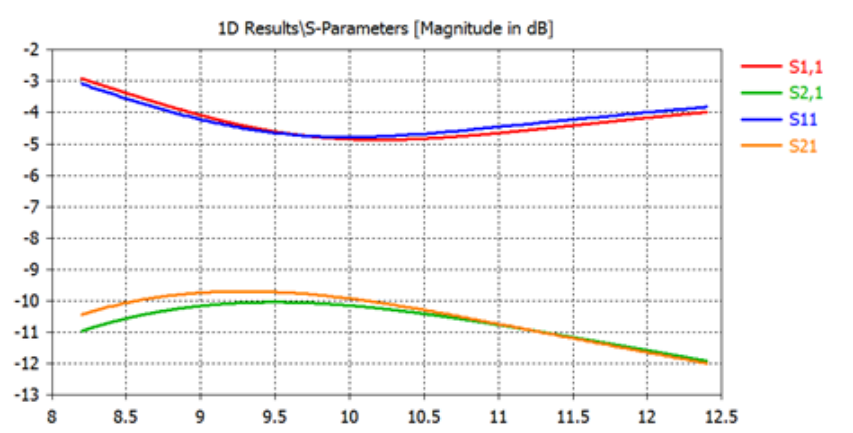

Fig. 4. The module of the reflection and transmission coefficients of the ПКМ-35Ж material samples: red and green curves - calculated, blue and orange curves - measured.
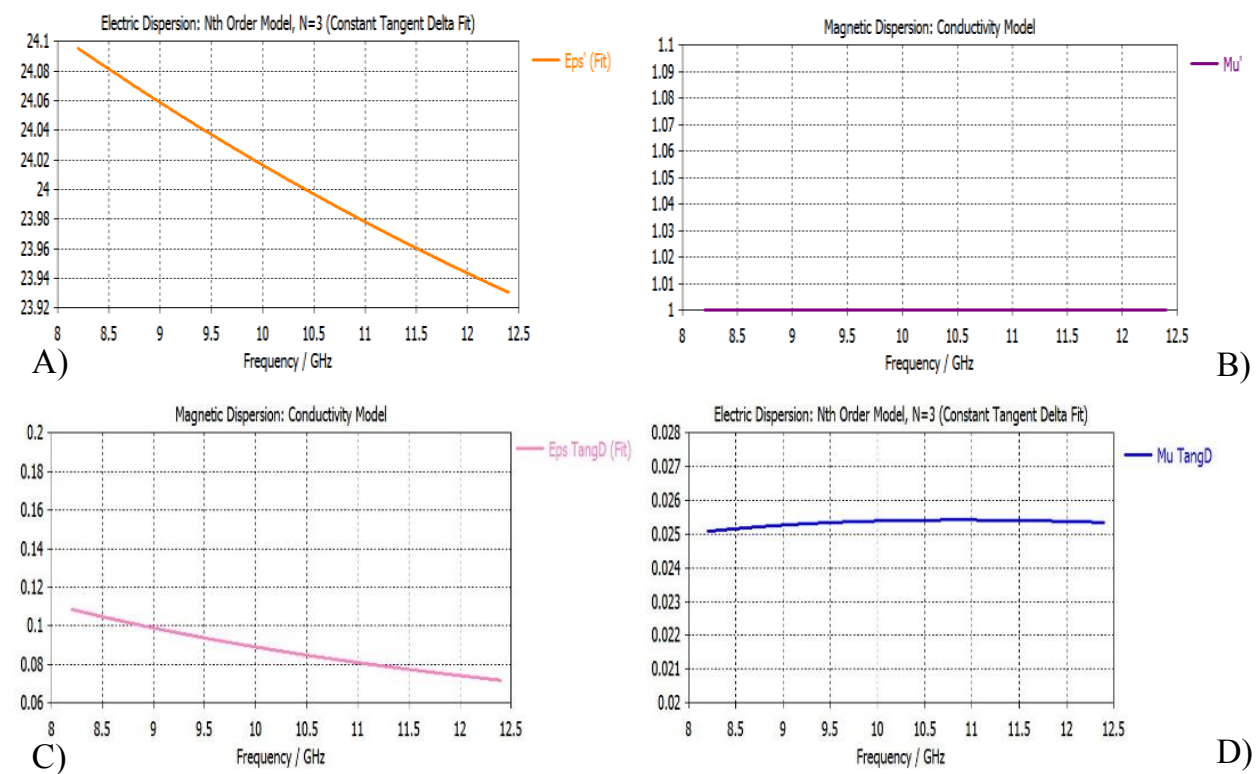

Fig. 5. A) permittivity; B) dielectric loss tangent; C)permeability; D)magnetic loss tangent. 

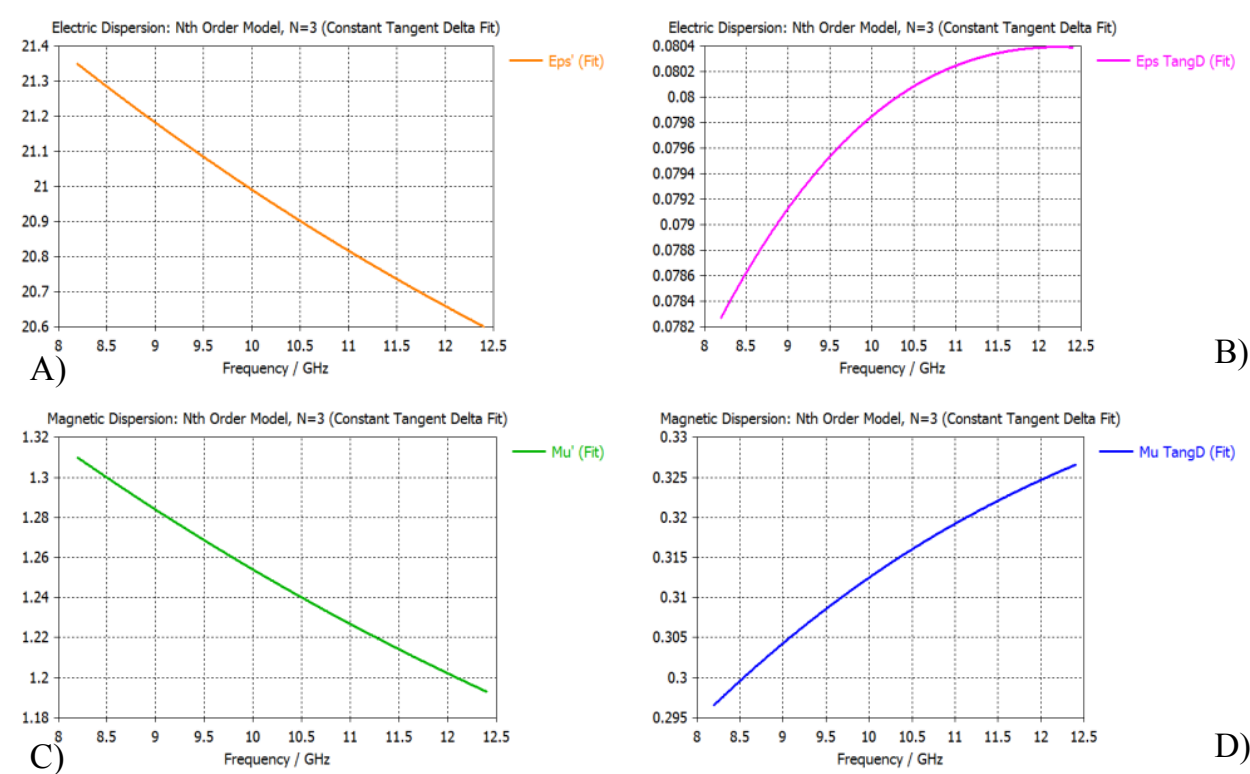

Fig. 6. Electromagnetic parameters of ПКМ-35Ж ceramics: A)permittivity; B) dielectric loss tangent; C)permeability; D)magnetic loss tangent.

\section{Conclusion}

The developed method for measuring electrodynamic characteristics of materials with high permittivity allows to obtain complete information about the material in a wide frequency band with a relative error of $2 \%$ regardless of the gap size (provided that the gap is accurately measured).

\section{References}

1. M. Afsar, J. Birch, and R. Clarke, The measurement of the properties of materials, Proc. IEEE, v. 74, 1 (1986)

2. V. Shestopalov, K. Yatsuk, Methods for measuring dielectric permeability of matter at ultrahigh frequency, Advanced in physical Science, v. 4 (1961)

3. A. Hippel, Dielectric and waves (M. Gosenergoizdat 1959)

4. Agilent Techologies, Fundamentals of measurement of dielectric properties of materials. Notes on application (2010)

5. A. Nicolson, G. Ross, Measurement of the intrinsic properties of materials by timedomain techniques, IEEE Trans. on Instrum. Meas., v. 19(4), pp. $377-382$ (1970)

6. W. Weir Automatic measurement of complex dielectric constant and permeability at microwave frequencies, Proceedings of the IEEE, v. 62(1), pp. 33-36 (1974)

7. L. Chen, C. Ong and C. Neo, Microwave electronics. Measurements and materials characterization (National University of Singapore 2004)

8. B. Bundy, Methods optimizations (M. Radio and Communication 1988) 